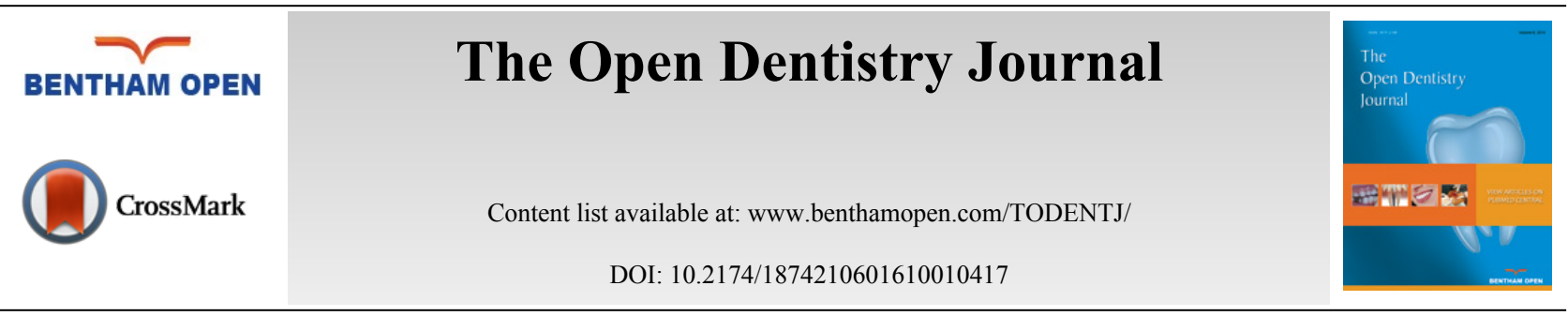

LETTER

\title{
Need of Oral Pre Cancer Awareness Initiatives in India
}

\author{
Stuti Bhargava \\ Department of Oral Medicine and Radiology, VSPM Dental College and Research Centre, Nagpur, India
}

The incidence and prevalence of oral precancerous conditions and lesions is very high in various parts of India, especially Central India. Most commonly seen oral pre cancerous conditions here include oral submucous fibrosis (OSMF), leukoplakia, oral lichen planus (OLP) and erythroleukoplakia. Facts and figures about these conditions such as aetiology, clinical features, signs and symptoms, staging, rate of transformation from precancer to cancer and high number of affected population are well documented in literature. The health care fraternity, chiefly the dentists, oncologists, ENT specialists and even physicians also have in depth knowledge of these conditions. However general population remains largely unaware about the concept of oral precancer. Often individuals with precancer even notice the alterations, such as reduced mouth opening in OSMF, but they are not aware about the causes and consequences of these changes. Awareness about oral precancers causes and features can be very helpful in prevention, control and early diagnosis of oral cancer.

India has been proactive in initiating many campaigns for public awareness, habit cessation along with legislations against availability of causative agents of oral cancer. These campaigns mainly advise against the use of alcohol, tobacco and tobacco preparations such as cigarettes, bidi, gutkha etc.; by drawing attention to the fact that a frequent consequence of such addictive habits is oral cancer. The warnings issued by means of televised infomercials, hoardings and on commercial packaging's of tobacco products mostly depict graphic images of advanced stages of cancerous lesions, however not much of an emphasis is laid on the possibility of occurrence of oral precancer and its appearances. Similarly somewhat inadequate warnings are issued regarding usage of many other potentially harmful products and preparations, which are widely available and consumed by the population. An important example of such prospectively harmful preparation is -Kharra -a local product commonly consumed in Central India which is a smokeless, chewable combination of tobacco, beetle nut and lime. Kharra consumption is a remarkably frequent and regular addictive habit amongst precancer patients in central India. Other such products include beetle nut, arecanut, lime, catechu, preparations such as pan quid (beetle leaf), pan masala, jarda, khaini, hookah, snuff, naswar etc.

The general population is largely unaware about the plausible association of $\boldsymbol{k h a r r a}$, beetle nut and many such products with oral precancer and cancer. There are also many self-perceived notions and misconceptions the populace has about the use of these substances. Some of the common mistaken beliefs are: (a) Tobacco, beetle nut etc.; consumed regularly but in self perceived, small quantities are not harmful; rather they may be helpful in improving digestion, increasing concentration and even in pain relief (b) Only smoking is harmful and chewable forms do not posses any threat (c) these products may be harmful only in the old age and not the young (d) quantity of tobacco in kharra and pan quids is very less to cause any potential harm (e) beetle nut/arecanut and beetle leaf (pan) are used in many Hindu religious customs, so a great part of the population does not envision any probable disadvantages from consumption of these products. Other challenges in habit cessation include psychological dependence, withdrawal symptoms and unavailability of guidance for structured de addiction.

\footnotetext{
* Address correspondence to this author at the Department of Oral Medicine and Radiology, VSPM Dental College and Research Centre, Nagpur, India; Tel:+91 9096843907; E-mail: drstutibhargava@gmail.com
} 
If the population is made aware of the precancerous conditions and lesions and it may be very helpful in prevention, control and early diagnosis of oral cancer. Early detection and management of oral pre cancer and cancer results in a better prognosis. There is an alarming lack of awareness about oral precancer, due to this gap in knowledge the affected individuals do not seek treatment in the early stages of the disease [1].

Deducing from already established facts in the literature and from ongoing doctoral project, I would like to put forth certain suggestion which may be further helpful in enhancing the effectiveness of existing programs and implementation of newer policies to increase the awareness about oral pre cancer and thus prevent any eventual development of oral cancer;

1. The conditions under the umbrella of oral precancer should also have easy to use names in local vernacular languages instead of the currently used long and difficult to remember medical terminologies and nomenclature which are difficult for general population to follow.

2. Information about potentially harmful causative agents, products, preparations / mixtures, signs, symptoms, clinical features, self-examination methods and possible transformation of these precancerous conditions in to cancerous conditions should be highlighted for the population. Examples of such initiatives is the information being provided for conditions like diabetes, dengue, swine flu etc. which makes it easier for the patients to notice developments in the initial stages and seek help for the same.

3. Infomercials should not only highlight advanced stages of cancer as they mostly do at present but also draw attention towards precancerous conditions and their appearances.

4. Specially designed programs should be initiated for school going population, adolescents and young adults, as it is observed that, these age groups are vulnerable to addiction of aforementioned high risk products and they gradually progress from one product to another. e.g. Fennel chewer gradually starts consuming beetle nut then kharra, gutka and progresses to more concentrated preparations of smokeless or smoking forms of tobacco.

5. Professional establishments; where it is now a known fact that the employees tend to consume addictive products as a result of work stress should adopt policies such as no tobacco in the premises/on duty and should provide facilities for regular oral health checkup, cessation counseling and NRTs. Common examples of such vocations in India include factory workers, workers of night shifts, drivers of transport corporations etc.

6. Number of centers providing habit cessation, de addiction counseling facilities should be increased. Similarly number of telephonic hotlines providing information about the conditions and cessation should also be increased. Such avenues must be able to help users from all age groups and socio economic back grounds.

7. It is commonly observed that the dentists, physicians many times refrain from counseling or informing the individuals with oral precancer, users of tobacco, beetle nut etc. about impending dangers. Such counseling is a known to promote habit stoppage and thus further hazardous consequences. Health care workers must be trained in conducting such cessation sessions.

8. Attention must also be given to eradicate the misconceptions about usage of such products.

Awareness and knowledge about oral precancer and potentially malignant disorders and their risk factors is crucial for prevention and early detection of oral cancer [2]. There is lack of published data available on this aspect in the context of Indian population thus highlighting the need of further studies to be carried out in this direction.

\section{CONFLICT OF INTEREST}

The author confirms that this article content has no conflict of interest.

\section{ACKNOWLEDGEMENTS}

Declared none.

\section{REFERENCES}

[1] Ariyawardana A, Vithanaarachchi N. Awareness of oral cancer and precancer among patients attending a hospital in Sri Lanka. Asian Pac J Cancer Prev 2005; 6(1): 58-61.

[PMID: 15780034] 
[2] Formosa J, Jenner R, Nguyen-Thi M, Stephens C, Wilson C, Ariyawardana A. Awareness and knowledge of oral cancer and potentially malignant oral disorders among dental patients in Queensland. Asian Pac J Cancer Prev 2015; 16(10): 4429-34.

[http://dx.doi.org/10.7314/APJCP.2015.16.10.4429] [PMID: 26028109]

C) Stuti Bhargava1; Licensee Bentham Open

This is an open access article licensed under the terms of the Creative Commons Attribution-Non-Commercial 4.0 International Public License (CC BY-NC 4.0) (https://creativecommons.org/licenses/by-nc/4.0/legalcode), which permits unrestricted, non-commercial use, distribution and reproduction in any medium, provided the work is properly cited. 